\title{
Predictive Power of Bone Turnover Biomarkers to Estimate Bone Mineral Density after Kidney Transplantation with or without Denosumab: A post hoc Analysis of the POSTOP Study
}

\author{
Nadine Heimgartner ${ }^{a} \quad$ Nicole Graf $^{b}$ Diana Frey ${ }^{c} \quad$ Lanja Saleh $^{d}$ \\ Rudolf P. Wüthricha Marco Bonani ${ }^{a}$ \\ ${ }^{a}$ Division of Nephrology, University Hospital Zürich, Zürich, Switzerland; b graf Biostatistics, \\ Winterthur, Switzerland; 'Division of Rheumatology, University Hospital Zürich, Zürich, \\ Switzerland; dInstitute of Clinical Chemistry, University Hospital Zürich and University of \\ Zürich, Zürich, Switzerland
}

\section{Keywords}

Kidney transplantation - Bone mineral metabolism - Denosumab - Biomarkers of bone turnover

\begin{abstract}
Background: Low bone mineral density (BMD) represents a major risk factor for bone fractures in patients with chronic kidney disease (CKD) as well as after kidney transplantation. However, modalities to solidly predict patients at fracture risk are yet to be defined. Better understanding of bone turnover biomarkers (BTMs) may close this diagnostic gap. This study strives to correlate BTMs to BMD in kidney transplant recipients. Methods: Changes in BTMs - procollagen type I N-terminal propeptide (P1NP), bone-specific alkaline phosphatase (BSAP), $\beta$-isomer of the $C$-terminal telopeptide of type I collagen, and urine deoxypyridinoline/ $\mathrm{Cr}$ - at the time of transplant and 3 months were correlated to changes in BMD measured by dualenergy X-ray absorptiometry at the time of transplant, 6 , and 12 months, respectively. Half of the collective was treated with denosumab twice yearly in addition to the standard treatment with calcium and vitamin D. Results: Changes in bone formation markers BSAP and P1NP within 3 months showed a significant negative correlation to changes in BMD at the hip within 6 months in denosumab-naïve patients. This correlation was abrogated by denosumab treatment. Conclusions: Changes in BSAP and P1NP showed promise in short-term prediction
\end{abstract}




\section{Kidney \\ Blood Pressure \\ Research}

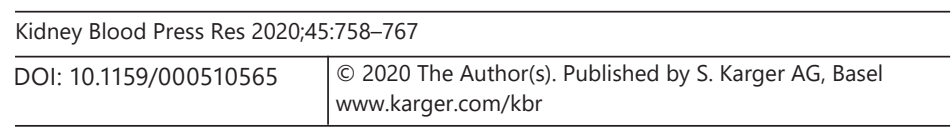

Heimgartner et al.: Biomarkers of Bone Turnover to Predict BMD after Kidney Transplantation

of BMD. We suggest further trials expanding on the knowledge of these BTMs with assessment of fracture risk, sequential measurements of BTMs within the first 6 months, and the additional use of computed tomography to assess BMD.

(C) 2020 The Author(s)

Published by S. Karger AG, Basel

\section{Introduction}

Chronic kidney disease (CKD) is accompanied by relevant changes in bone and mineral metabolism which can lead to renal osteodystrophy [1]. The high prevalence of bone disease in patients with CKD implicates that kidney transplant candidates often present with osteopenia or osteoporosis. The already low bone mineral density (BMD) may further decrease after transplantation, especially in the first year after transplantation and mainly because of glucocorticoids. They have a powerful detrimental effect on the skeleton by several mechanisms and are still a main pillar of immunosuppression after transplantation [2]. In addition, pre- and posttransplant hyperparathyroidism leads to a mechanical compromise of the bone [3]. Therapeutic amelioration of bone loss after kidney transplantation includes the supplementation of calcium and vitamin D [4,5]. Another controversially discussed treatment constitutes bisphosphonates, which indeed improve bone loss, but are giving cause to several concerns, including nephrotoxicity. Controversial data on fracture prevention in renal transplant recipients and the occurrence of atypical fractures in these patients impede their widespread application [6-14]. The RANKL antibody denosumab represents a newer strategy in preventing posttransplant bone loss, which had superior efficacy in improving BMD and preventing fractures in postmenopausal women with osteoporosis as compared to bisphosphonates $[15,16]$.

Dual-energy X-ray absorptiometry (DXA) scan represents the gold standard to diagnose osteoporosis and its severity, but it suffers from methodological limitations [17]. In contrast, bone turnover biomarkers (BTMs) can possibly identify patients with rapid bone loss $[18,19]$ and may assist in monitoring treatment efficacy before BMD decline becomes obvious by DXA. Ability of early BMD loss recognition with establishment of timely treatment may further reinforce patient compliance. Nevertheless, current expert consensus states that BTMs should not guide BMD surveillance solely but may monitor osteoporosis treatment efficacy. Widespread application is hampered by their biological variability [20-23] and controversial data of fracture prediction [24].

In the POSTOP trial by Bonani et al. [25], a total of 4 BTMs apart from the parathyroid hormone (PTH) were studied: 2 markers of bone formation, procollagen type I N-terminal propeptide (P1NP) and bone-specific alkaline phosphatase (BSAP), and 2 markers of bone resorption, $\beta$-isomer of the $\mathrm{C}$-terminal telopeptide of type I collagen ( $\beta$-CTX), and urine deoxypyridinoline/Cr (DPD). BTMs are not specific for a single bone disease and reflect the entire skeleton [26]. P1NP is a specific by-product of active osteoblasts. The extension peptide $\mathrm{P} 1 \mathrm{NP}$ is cleaved by enzymes at the end of the procollagen molecule during the formation of bone matrix [23]. It has been recommended by the Bone Marker Standards Working Group because of its low inter-individual variability [23] and its stability in serum at room temperature [27]. BSAP on the other hand is a specific product of bone osteoblasts as well but reflects enzymatic activity. Osteoid mineralization is mediated by alkaline phosphatase secreted by osteoblasts [23].

Most of the bone resorption markers are degradation products of bone collagen. The pyridinium cross-links DPD are a by-product during bone maturation and are released during the bone resorption process. The same holds true for the peptide $\beta$-CTX of the type I collagen molecule [23]. $\beta$-CTX surfaced as the marker of choice, although it has a large circadian vari- 


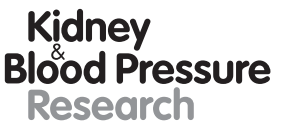

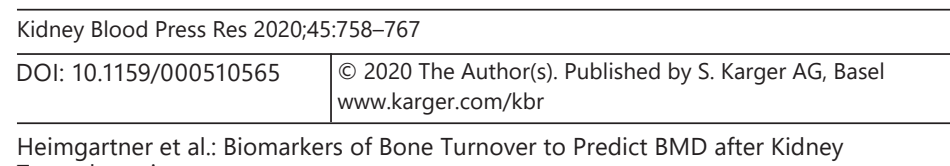

Heimgartner et
Transplantation

ation with the requirement of a morning fasting blood sample for accurate interpretation [23, 28].

The POSTOP study group was able to show an increase in BMD measured by DXA after twice-yearly denosumab in the first year after kidney transplantation. Moreover, a significant decrease of all studied BTMs was documented in the denosumab group [25]. This post hoc analysis of the POSTOP trial aims to investigate whether the changes in the BMD at the lumbar spine and total hip at 6 and 12 months can be predicted by the changes in BTMs at 3 months.

\section{Materials and Methods}

\section{Patients and Study Design}

This study is a post hoc analysis of the POSTOP trial by Bonani et al. [25] (Effect of TwiceYearly Denosumab on Prevention of Bone Mineral Density Loss in De Novo Kidney Transplant Recipients: a Randomized Controlled Trial). The POSTOP trial was a 1-year prospective singlecenter, randomized, parallel-group, open-label clinical trial in de novo kidney transplant recipients. The study was approved by the local ethics steering committee, conformed to the Declaration of Helsinki and Istanbul, and has been registered with ClinicalTrials.gov (NCT01377467).

Included patients were adult woman and man with end-stage renal disease (ESRD). Transplantation took place up to 28 days previously with a kidney allograft and patients were treated with standard immunosuppression, that is, calcineurin antagonist, mycophenolate, and corticosteroids. Patients had to be excluded if there was evidence of a poor or unstable graft function $(\mathrm{Cr}>200 \mu \mathrm{mol} / \mathrm{L})$, severe osteoporosis ( $T$-score below -4.0 ), severe hyper or hypoparathyroidism (iPTH $>800$ or $<10 \mathrm{ng} / \mathrm{L}$ ), and hypo or hypercalcemia (total calcium $<1.8$ or $>2.7 \mathrm{mmol} / \mathrm{L}$ ). Randomization followed a 1:1 protocol to the denosumab group (60 $\mathrm{mg}$ denosumab s.c. at baseline and after 6 months) or no treatment. The investigators of the biomarker measurements were masked to allocation. Serum and urine samples were collected from all patients at baseline and after 3, 6, and 12 months. The analyzed BTMs included P1NP, BSAP, $\beta$-CTX, and DPD.

\section{Laboratory Measurements}

Standard laboratory techniques were used to assess levels of BTMs as prior described [29]. The eGFR was calculated according to the CKD-EPI formula.

\section{Dual-Energy X-Ray Absorptiometry}

Measurements of BMD were obtained by DXA at baseline and at 6 and 12 months in all patients. Two anatomic sites were assessed: first, the lumbar spine constituting of averages between vertebrae L1 to L4 and, second, the proximal femur (total hip). The examination was conducted with a Hologic QDR 4500 A device, and the results were expressed as a relative value in the number of SD below the expected normal gender- and ethnicity-adjusted BMD values ( $T$-Score).

\section{Statistical Analyses}

All data were analyzed using R version 3.6.1. Percentage change of BMD was calculated between baseline and visits at 6 and 12 months; absolute change was computed for all biomarkers between baseline and visit at 3 months. Spearman's rho between biomarkers and BMD was appraised between the control and denosumab group with the package "Hmisc" [30], and all $p$ values were Holm corrected within treatment groups. The figures were plotted with the package "ggplot2" [31]. 
Heimgartner et al.: Biomarkers of Bone Turnover to Predict BMD after Kidney

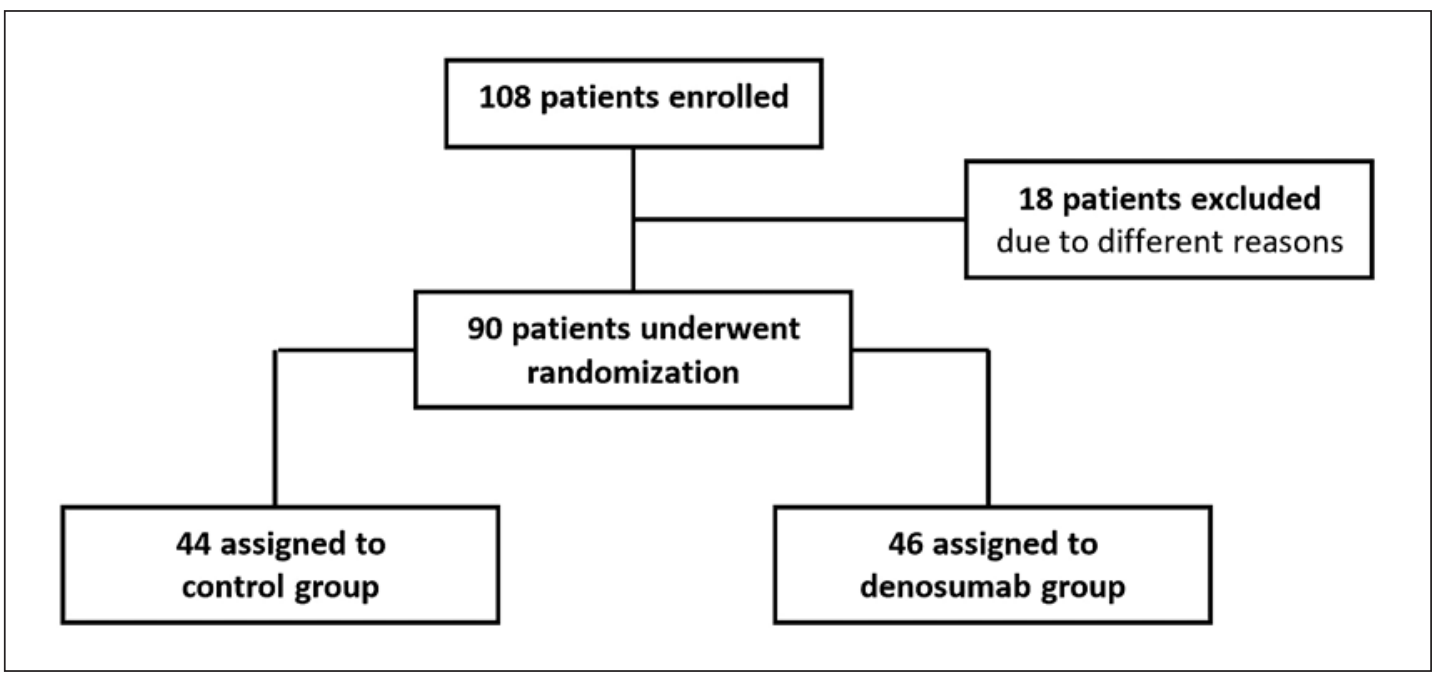

Fig. 1. Study flow chart.

Table 1. Baseline characteristics of patients

\begin{tabular}{lcc}
\hline & $\begin{array}{c}\text { Control } \\
(n=44)\end{array}$ & $\begin{array}{c}\text { Denosumab } \\
(n=46)\end{array}$ \\
\hline Age, years & $49.0 \pm 12.9$ & $50.0 \pm 14.0$ \\
Men, $n$ (\%) & $22(50.0)$ & $35(76.1)$ \\
White ethnicity, $n$ (\%) & $42(95.5)$ & $44(95.7)$ \\
Repeat transplantation, $n$ (\%) & $7(15.9)$ & $7(15.2)$ \\
BMD and T-scores & & \\
$\quad$ Total lumbar spine BMD, g/cm ${ }^{2}$ & $0.934 \pm 0.129$ & $1.002 \pm 0.139$ \\
Total lumbar spine $T$-score & $-1.27 \pm 1.15$ & $-0.67 \pm 1.25$ \\
Total hip BMD, g/cm ${ }^{2}$ & $0.847 \pm 0.092$ & $0.925 \pm 0.132$ \\
Total hip T-score & $-1.05 \pm 0.65$ & $-0.59 \pm 0.97$ \\
Osteopenic patients, $n$ (\%) & $25(56.8)$ & $16(34.8)$ \\
Osteoporotic patients, $n$ (\%) & $6(13.6)$ & $3(6.5)$ \\
P1NP, $\mu \mathrm{g} / \mathrm{L}$ & $75.3 \pm 44.4$ & $66.6 \pm 51.6$ \\
BSAP, $\mu \mathrm{g} / \mathrm{L}$ & $15.7 \pm 13.9$ & $13.5 \pm 14.8$ \\
$\beta$-CTX, $\mu \mathrm{g} / \mathrm{L}$ & $0.8 \pm 0.4$ & $0.6 \pm 0.3$ \\
DPD, nmol/mmol & $8.2 \pm 2.7$ & $7.2 \pm 3.0$ \\
PTH, ng/L & $147.6 \pm 137.1$ & $163.2 \pm 153.5$ \\
\hline
\end{tabular}

Data are mean $\pm \mathrm{SD}$, or $n(\%)$. PTH, parathyroid hormone; BSAP, bone-specific alkaline phosphatase; P1NP, procollagen-1 N-terminal propeptide; $\beta$-CTX, $\beta$-isomer of C-terminal telopeptide of type I collagen; DPD, urine deoxypyridinoline/Cr.

\section{Results}

A total of 90 kidney transplant recipients with a functioning graft were included (Fig. 1). After transplantation, patients were randomized to denosumab group $(60 \mathrm{mg}$ s.c. at baseline and at 6 months, $n=46)$ or control group receiving no treatment $(n=44)$. Baseline characteristics were balanced between the 2 groups excepting gender with a majority of men in the denosumab group resulting in a higher baseline BMD (Table 1). The mean age of the patients was $49.0 \pm 12.9$ years, roughly two-third were male (63.3\%). Most study participants in both 
Table 2. Time-dependent BMD and bone markers' behavior

\begin{tabular}{|c|c|c|c|c|}
\hline Parameter & Baseline & 3 months & 6 months & 12 months \\
\hline \multicolumn{5}{|l|}{ Control $(n=44)$} \\
\hline Total lumbar spine BMD, $\mathrm{g} / \mathrm{cm}^{2}$ & $0.934 \pm 0.129$ & & $0.922 \pm 0.135$ & $0.933 \pm 0.136$ \\
\hline Total hip BMD, $\mathrm{g} / \mathrm{cm}^{2}$ & $0.847 \pm 0.092$ & & $0.847 \pm 0.095$ & $0.854 \pm 0.100$ \\
\hline $\mathrm{P} 1 \mathrm{NP}, \mu \mathrm{g} / \mathrm{L}$ & $75.3 \pm 44.4$ & $106.9 \pm 89.9$ & & \\
\hline BSAP, $\mu \mathrm{g} / \mathrm{L}$ & $15.7 \pm 13.9$ & $15.9 \pm 10.6$ & & \\
\hline$\beta-\mathrm{CTX}, \mu \mathrm{g} / \mathrm{L}$ & $0.8 \pm 0.4$ & $0.6 \pm 0.4$ & & \\
\hline $\mathrm{DPD}, \mathrm{nmol} / \mathrm{mmol}$ & $8.2 \pm 2.7$ & $7.2 \pm 3.5$ & & \\
\hline PTH, ng/L & $147.6 \pm 137.1$ & $113.0 \pm 84.5$ & & \\
\hline \multicolumn{5}{|l|}{ Denosumab $(n=46)$} \\
\hline Total lumbar spine BMD, $\mathrm{g} / \mathrm{cm}^{2}$ & $1.002 \pm 0.139$ & & $1.033 \pm 0.140$ & $1.054 \pm 0.140$ \\
\hline Total hip BMD, $\mathrm{g} / \mathrm{cm}^{2}$ & $0.925 \pm 0.132$ & & $0.941 \pm 0.132$ & $0.955 \pm 0.130$ \\
\hline $\mathrm{P} 1 \mathrm{NP}, \mu \mathrm{g} / \mathrm{L}$ & $66.6 \pm 51.6$ & $40.8 \pm 34.8$ & & \\
\hline BSAP, $\mu \mathrm{g} / \mathrm{L}$ & $13.5 \pm 14.8$ & $8.6 \pm 7.2$ & & \\
\hline$\beta-\mathrm{CTX}, \mu \mathrm{g} / \mathrm{L}$ & $0.6 \pm 0.3$ & $0.1 \pm 0.1$ & & \\
\hline $\mathrm{DPD}, \mathrm{nmol} / \mathrm{mmol}$ & $7.2 \pm 3.0$ & $5.0 \pm 1.6$ & & \\
\hline PTH, ng/L & $163.2 \pm 153.5$ & $167.2 \pm 173.6$ & & \\
\hline
\end{tabular}

Data are mean \pm SD. PTH, parathyroid hormone; BSAP, bone-specific alkaline phosphatase; P1NP, procollagen-1 N-terminal propeptide; $\beta$-CTX, $\beta$-isomer of C-terminal telopeptide of type I collagen; DPD, urine deoxypyridinoline/Cr.

Table 3. Correlation of bone formation and resorption markers (absolute change) after 3 months and BMD measured at lumbar spine and hip (percentage change) after 6 and 12 months of follow-up

\begin{tabular}{|c|c|c|c|c|c|c|c|}
\hline \multirow[b]{2}{*}{ P1NP control } & \multicolumn{2}{|c|}{$\begin{array}{l}\text { BMD lumbar spine, } \\
6 \text { months }\end{array}$} & \multicolumn{2}{|c|}{$\begin{array}{l}\text { BMD hip, } \\
6 \text { months }\end{array}$} & $\begin{array}{l}\text { BMD lumbar } \\
\text { spine, } 12 \text { months }\end{array}$ & \multicolumn{2}{|c|}{$\begin{array}{l}\text { BMD hip, } \\
12 \text { months }\end{array}$} \\
\hline & -0.388 & {$[0.167]$} & $-0.484^{a}$ & {$[0.022]$} & $-0.010 \quad[1.000]$ & -0.214 & [1.000] \\
\hline P1NP denosumab & -0.293 & {$[0.966]$} & -0.046 & {$[1.000]$} & $-0.240 \quad[1.000]$ & +0.042 & [1.000] \\
\hline BSAP control & $-0.506^{a}$ & {$[0.013]$} & $-0.476^{\mathrm{a}}$ & {$[0.026]$} & $-0.075[1.000]$ & -0.238 & {$[1.000]$} \\
\hline BSAP denosumab & -0.258 & [1.000] & -0.035 & [1.000] & $-0.128 \quad[1.000]$ & -0.026 & {$[1.000]$} \\
\hline $\mathrm{DPD}^{\mathrm{b}}$ control & -0.476 & {$[0.075]$} & -0.296 & [1.000] & $-0.212 \quad[1.000]$ & -0.160 & [1.000] \\
\hline $\mathrm{DPD}^{\mathrm{b}}$ denosumab & -0.363 & {$[0.561]$} & -0.209 & [1.000] & $-0.376[0.524]$ & -0.156 & [1.000] \\
\hline$\beta$-CTX control & -0.298 & [0.769] & -0.408 & {$[0.116]$} & $-0.040[1.000]$ & -0.121 & [1.000] \\
\hline$\beta$-CTX denosumab & -0.102 & {$[1.000]$} & -0.104 & [1.000] & $-0.115[1.000]$ & -0.203 & [1.000] \\
\hline PTH control & +0.129 & [1.000] & +0.103 & {$[1.000]$} & $-0.102 \quad[1.000]$ & +0.010 & [1.000] \\
\hline PTH denosumab & +0.076 & {$[1.000]$} & +0.162 & {$[1.000]$} & $-0.330[0.627]$ & -0.082 & [1.000] \\
\hline
\end{tabular}

Data depicted as Spearman's rho [ $p$ value]. P1NP, procollagen-1 N-terminal propeptide; BSAP, bonespecific alkaline phosphatase; DPD, urine deoxypyridinoline/Cr; $\beta$-CTX, $\beta$-isomer of C-terminal telopeptide

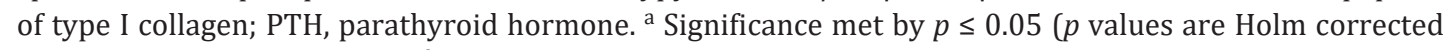
within the treatment group). ${ }^{\mathrm{b}}$ DPD had up to $15 \%$ missing values and in consequence even moderate correlations did not prove to be significant.

groups were Caucasian (95.6\%) and had been transplanted for the first time (84.4\%). All patients were prescribed standard treatment for posttransplant CKD-MBD consisting of calcium (control group $619 \pm 475 \mathrm{mg}$ q.d., denosumab group $756 \pm 609 \mathrm{mg}$ q.d.) and vitamin D supplementation (control group 1,400 \pm 1,295 IU q.d., denosumab group 1,526 $\pm 946 \mathrm{IU}$ q.d.). During the study, 2 patients in the denosumab group received cinacalcet. No other medi- 
Heimgartner et al.: Biomarkers of Bone Turnover to Predict BMD after Kidney

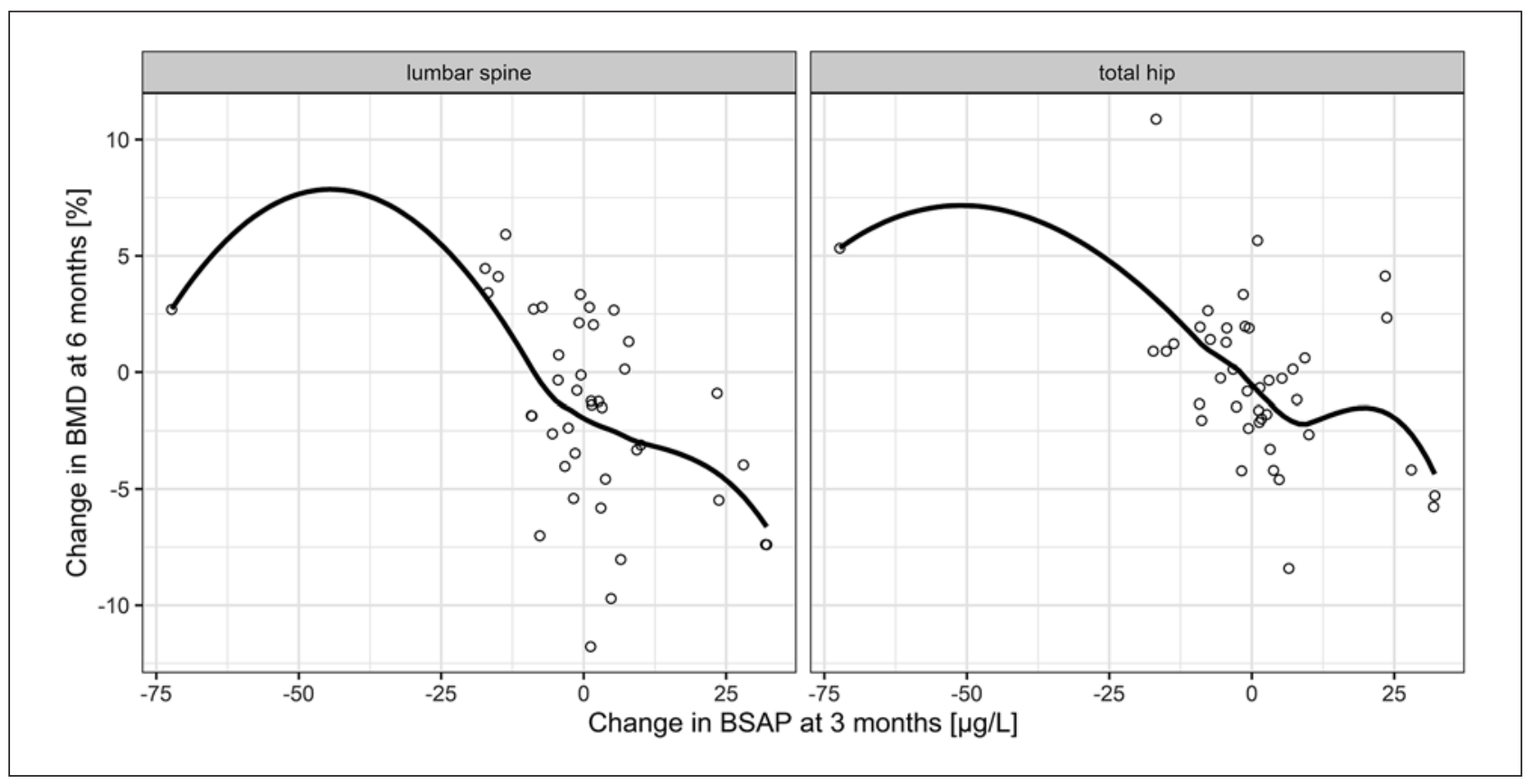

Fig. 2. Correlation of BSAP and BMD in denosumab-naïve patients. Dots show the absolute change in BSAP at 3 months versus the percentage change in BMD at 6 months and the line shows the average trend by using locally weighted regression (loess) with a span (degree of smoothing) of 0.85 . Spearman's rho was -0.476 for total hip and -0.506 for lumbar spine. BSAP, bone-specific alkaline phosphatase; BMD, bone mineral density.

cations with influence on the bone metabolism were used. At baseline, $10 \%$ of the patients were osteoporotic and $45 \%$ were osteopenic.

\section{The Bone Formation Markers: BSAP and P1NP}

Descriptive values of the bone formation markers at baseline as well as 3 months later are shown in Table 2. Change in levels of BSAP and P1NP after 3 months inversely correlated with the variation of BMD of the hip after 6 months in the control group. This was also seen for BSAP at lumbar spine. However, significance was not met for any site or biomarker in the denosumab group. Neither was any predictive value documented for the longer observation period over 12 months (Table 3; Fig. 2, 3).

The Bone Resorption Markers: $\beta$-CTX, DPD, and PTH

Descriptive values of bone resorption markers at baseline as well as 3 months later are shown in Table 2. None of the investigated bone resorption markers showed any significant correlation to change of BMD. This holds true for both control and denosumab group patients, as well as for both studied sites and time frames (Table 3).

\section{Discussion}

Renal osteodystrophy is a prevalent disorder in CKD, causing higher morbidity with reduction in quality of life and higher mortality [32]. After kidney transplantation, several additional detrimental factors reduce BMD on top of the pre-existing damage [3]. Hence, the 
Heimgartner et al.: Biomarkers of Bone Turnover to Predict BMD after Kidney

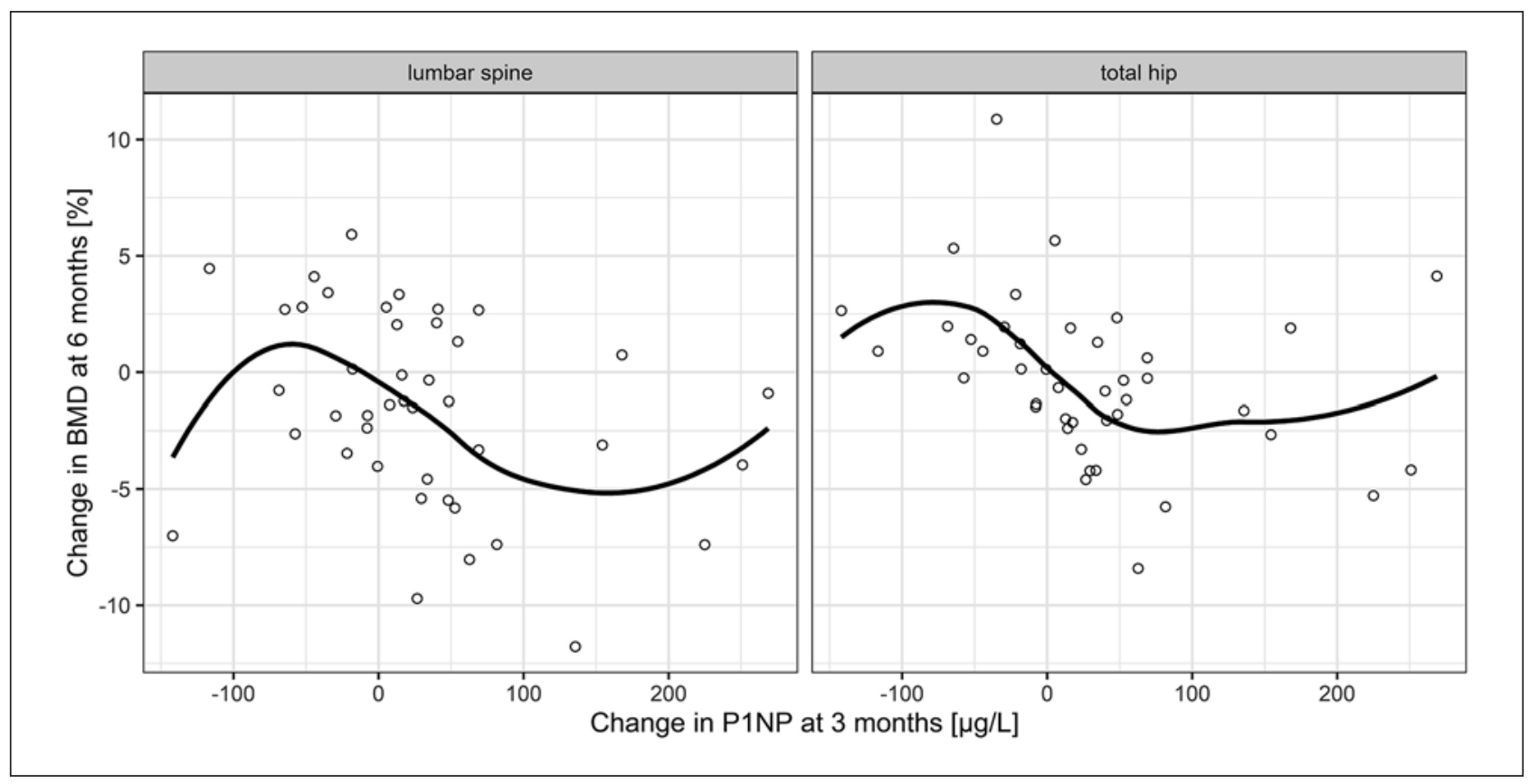

Fig. 3. Correlation of P1NP and BMD in denosumab-naïve patients. Dots show the absolute change in P1NP at 3 months versus the percentage change in BMD at 6 months and the line shows the average trend by using locally weighted regression (loess) with a span (degree of smoothing) of 0.85 . Spearman's rho was -0.484 for total hip and -0.388 for lumbar spine. P1NP, procollagen-1 N-terminal propeptide; BMD, bone mineral density.

prevention of osteoporosis or at least maintenance of BMD constitutes a very important role in posttransplantation management.

Loss of BMD is most rapid in the first 6-12 months after kidney transplantation [13]; therefore, early therapy strategies with denosumab, for example, are paramount [25]. Predictive markers of future BMD decline are necessary to best select patients in need of constant monitoring and aggressive treatment. This post hoc analysis aims to shed light on whether BTMs may be able to close this gap in diagnostics.

The current study documented a moderate negative correlation in denosumab-naïve patients in both studied bone formation markers after 3 months and BMD measured at the hip after 6 months. In contrast to P1NP, this finding holds true for BSAP and change of BMD at lumbar spine in the same time frame. No predictive value was documented for the studied bone resorption markers.

In a recent study by Keronen et al. [33], no correlation between the studied BTMs, that is, PTH, BSAP, osteoclastin, and BMD or bone histomorphometric findings was found in kidney-transplanted and dialysis patients, although bone formation and mineralization declined in the late posttransplant period. This finding of declining BMD is supported by similar data from an observational, prospective study by Evenepoel et al. [34]. Our analysis documented failure of BMD prediction for the 2 additional bone resorption markers DPD and $\beta$-CTX. In contrast to their findings, our study showed a moderate negative correlation between BSAP and BMD development. Furthermore, the intervals from baseline to the followup measurement of BTMs were 1 and 2 years, respectively. In the longer period after transplantation, that is, 12 months and beyond, all of the analyzed BTMs in our study equally failed to show a correlation. Strikingly, this emphasizes the importance of short-term predictors of 


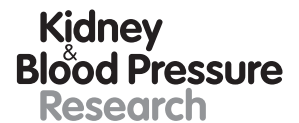

Kidney
Blood Pressure
Research

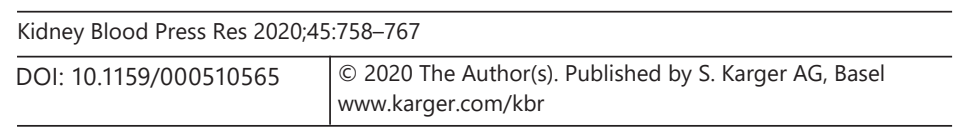

Heimgartner et al.: Biomarkers of Bone Turnover to Predict BMD after Kidney Transplantation

BMD development as documented in our study with the possibility of timely drug intervention in vulnerable patients.

Contrary to kidney transplantation, several valuable studies on BTMs in CKD patients are available. Varying patient cohorts, incomparable methods, and inconsistent results impede direct correlation to this study: In a trial focusing on all stages of CKD by Nickolas et al. [35], the studied BTMs failed to forecast changes in BMD measured by DXA. Interestingly, the study documented a correlation between the elevation of BTMs, including PTH and cortical deterioration assessed by high-resolution peripheral quantitative computed tomography at radius and tibia. This finding was confirmed in a dialysis collective by Malluche et al. [19] Additionally, they were able to document a predictive value of P1NP on bone gain in the spine by assessment with quantitative computed tomography. In patients with CKD, application of computed tomography for the assessment of BMD may catch momentum in the future due to mismeasurements using DXA in this population [19, 36-38]. The missing correlation of BTMs and lumbar spine BMD as compared to total hip in our data may be attributed to an accuracy error of DXA due to extensive calcification of the aorta in kidney transplant recipients [17].

Furthermore, contemporary treatment options for the mitigation of BMD loss also impact the levels of BTMs. Treatment of postmenopausal women with denosumab revealed a larger decrease of CTX and P1NP than those with alendronate [39]. This matches with the observation of a decrease of BTMs under denosumab treatment as compared to placebo control in kidney-transplanted patients [25]. Our data obtained from denosumab-treated patients failed to establish a correlation between BMD at any given time or site and $\beta$-CTX or P1NP. Brown et al. showed a low point of CTX under denosumab treatment as early as 1 month after application [39]. It may be hypothesized that a correlation of BMD and $\beta$-CTX may have been missed due to the delayed measurement after 3 months in our study design. However, limited comparability of osteoporosis in postmenopausal women and renal osteodystrophy may constitute an important caveat.

Our study had several limitations: First, there was a marginal imbalance in patient characteristics between the two groups. Second, lack of bone biopsies prohibited correlation of histomorphometric findings to BTM levels. Furthermore, assessment of BMD was only done by DXA, and no second modality such as high-resolution peripheral quantitative computed tomography was performed. Last but not least, the follow-up period and sample size did not allow for an investigation of fracture risk, which would represent the most relevant end point from a clinician's perspective.

In conclusion, we found a negative correlation between the early change of P1NP as well as BSAP and development of BMD at the hip after 6 months of kidney transplantation. This effect was abrogated by denosumab treatment. Among many factors leading to challenging comparability of data, the heterogeneity of renal osteodystrophy itself [1], the high preanalytic and biologic variability of BTMs [23], and the differences of measurement modalities are paramount. Further investigation is warranted to clarify whether BTM-based estimation of BMD translates into a correctly assessed risk of fracture and, in the long run, into biochemically guided individual treatment options. We suggest further studies with the four aforementioned BTMs, early sequential measurements within the first 6 months of treatment, and a broadening of diagnostic modalities with computed tomography. Last, understanding of both changes in microscopic bone structure and biochemical turnover is of tremendous importance to attempt sound prediction of skeleton health in patients with renal insufficiency. Therefore, as of now, we concur with current expert consensus to refrain from BMD prediction solely by BTMs. Moreover, the increased use of denosumab in kidney transplant recipients may further complicate BTM interpretation. 


\section{Kidney \\ Blood Pressure \\ Research}

\begin{tabular}{l|l}
\hline Kidney Blood Press Res 2020:45:758-767 \\
\hline DOI: 10.1159/000510565 & $\begin{array}{l}\text { @ 2020 The Author(s). Published by S. Karger AG, Basel } \\
\text { www.karger.com/kbr }\end{array}$ \\
\hline
\end{tabular}

Heimgartner et al.: Biomarkers of Bone Turnover to Predict BMD after Kidney Transplantation

\section{Statement of Ethics}

The POSTOP study, precursor of this post hoc analysis, was approved by the local ethics steering committee, conformed to the Declaration of Helsinki and Istanbul, and has been registered with Clinical Trials.gov (NCT01377467). Subjects (or their parents or guardians) gave their written informed consent.

\section{Conflict of Interest Statement}

The authors of this manuscript have conflicts of interest to disclose as described by Kidney and Blood Pressure Research: R.P.W. reports personal fees from Amgen, outside the submitted work. N.G. reports personal fees from Boehringer Ingelheim $\mathrm{GmbH}$, medac GmbH, and Bayer, outside the submitted work. A part of the study drug was obtained free of charge by Amgen Switzerland AG. The other authors have no conflicts of interest to disclose.

\section{Funding Sources}

This clinical trial was funded by the University Hospital Zürich and the University of Zürich.

\section{Author Contributions}

N.H. contributed to the interpretation of data and drafting of the manuscript. N.G. led the statistical analysis and revised the manuscript. D.F. and L.S. contributed to data analysis and revised the manuscript. R.P.W. and M.B. contributed to the conception and the design of the study, interpretation of data, and revised the manuscript. All authors approved the final version of the article and agree to be accountable for all aspects of this work.

\section{References}

1 Malluche HH, Monier-Faugere MC, Herberth J. Bone disease after renal transplantation. Nat Rev Nephrol. 2010 Jan;6(1):32-40.

2 Issue S. KDIGO clinical practice guideline for the care of kidney transplant recipients. Am J Transplant. 2009; 9:S1-155.

3 Cunningham J. Posttransplantation bone disease. Transplantation. 2005 Mar;79(6):629-34.

4 Josephson MA, Schumm LP, Chiu MY, Marshall C, Thistlethwaite JR, Sprague SM. Calcium and calcitriol prophylaxis attenuates posttransplant bone loss. Transplantation. 2004 Oct; 78(8):1233-6.

5 De Sévaux RG, Hoitsma AJ, Corstens FH, Wetzels JF. Treatment with vitamin D and calcium reduces bone loss after renal transplantation: a randomized study. J Am Soc Nephrol. 2002 Jun;13(6):1608-14.

6 Grotz W, Nagel C, Poeschel D, Cybulla M, Petersen KG, Uhl M, et al. Effect of ibandronate on bone loss and renal function after kidney transplantation. J Am Soc Nephrol. 2001 Jul;12(7):1530-7.

7 Jeffery JR, Leslie WD, Karpinski ME, Nickerson PW, Rush DN. Prevalence and treatment of decreased bone density in renal transplant recipients: a randomized prospective trial of calcitriol versus alendronate. Transplantation. 2003 Nov;76(10):1498-502.

8 Torregrosa JV, Fuster D, Gentil MA, Marcen R, Guirado L, Zarraga S, et al. Open-label trial: effect of weekly risedronate immediately after transplantation in kidney recipients. Transplantation. 2010 Jun;89(12):1476-81.

9 Smerud KT, Dolgos S, Olsen IC, Åsberg A, Sagedal S, Reisæter AV, et al. A 1-year randomized, double-blind, placebo-controlled study of intravenous ibandronate on bone loss following renal transplantation. Am J Transplant. 2012 Dec;12(12):3316-25.

10 Conley E, Muth B, Samaniego M, Lotfi M, Voss B, Armbrust M, et al. Bisphosphonates and bone fractures in long-term kidney transplant recipients. Transplantation. 2008 Jul;86(2):231-7.

11 Stein EM, Ortiz D, Jin Z, McMahon DJ, Shane E. Prevention of fractures after solid organ transplantation: a metaanalysis. J Clin Endocrinol Metab. 2011 Nov;96(11):3457-65. 


\begin{tabular}{l|l}
\hline Kidney Blood Press Res 2020;45:758-767 \\
\hline DOI: 10.1159/000510565 & $\begin{array}{l}\text { (c) 2020 The Author(s). Published by S. Karger AG, Basel } \\
\text { www.karger.com/kbr }\end{array}$ \\
\hline
\end{tabular}

Heimgartner et al.: Biomarkers of Bone Turnover to Predict BMD after Kidney

Transplantation

12 Park W, Lee SH, Park KR, Rho SH, Chung WY, Kim HJ. Characteristics of bisphosphonate-related osteonecrosis of the jaw after kidney transplantation. J Craniofac Surg. 2012 Sep;23(5):e510.

13 Ebeling PR. Approach to the patient with transplantation-related bone loss. J Clin Endocrinol Metab. 2009; 94(5):1483-90.

14 Marques IDB, Araújo MJCLN, Graciolli FG, dos Reis LM, Pereira RMR, Alvarenga JC, et al. A randomized trial of zoledronic acid to prevent bone loss in the first year after kidney transplantation. J Am Soc Nephrol. 2019 Feb; 30(2):355-65.

15 Cummings SR, San Martin J, McClung MR, Siris ES, Eastell R, Reid IR, et al. Denosumab for prevention of fractures in postmenopausal women with osteoporosis. N Engl J Med. 2009 Aug;361(8):756-65.

16 Kendler DL, Roux C, Benhamou CL, Brown JP, Lillestol M, Siddhanti S, et al. Effects of denosumab on bone mineral density and bone turnover in postmenopausal women transitioning from alendronate therapy. J Bone Miner Res. 2010 Jan;25(1):72-81.

17 Kanis JA, McCloskey EV, Johansson H, Oden A, Melton LJ, Khaltaev N. A reference standard for the description of osteoporosis. Bone. 2008 Mar;42(3):467-75.

18 Ross PD, Knowlton W. Rapid bone loss is associated with increased levels of biochemical markers. J Bone Miner Res. 1998 Feb;13(2):297-302.

19 Malluche HH, Davenport DL, Cantor T, Monier-Faugere MC. Bone mineral density and serum biochemical predictors of bone loss in patients with CKD on dialysis. Clin J Am Soc Nephrol. 2014 Jul;9(7):1254-62.

20 Fisher A, Fisher L, Srikusalanukul W, Smith PN. Bone turnover status: classification model and clinical implications. Int J Med Sci. 2018;15(4):323-38.

21 Bergmann P, Body JJ, Boonen S, Boutsen Y, Devogelaer JP, Goemaere S, et al. Evidence-based guidelines for the use of biochemical markers of bone turnover in the selection and monitoring of bisphosphonate treatment in osteoporosis: a consensus document of the Belgian Bone Club. Int J Clin Pract. 2009 Jan;63(1):19-26.

22 Greenblatt MB, Tsai JN, Wein MN. Bone turnover markers in the diagnosis and monitoring of metabolic bone disease. Clin Chem. 2017 Feb;63(2):464-74.

23 Vasikaran S, Eastell R, Bruyère 0 , Foldes AJ, Garnero P, Griesmacher A, et al. Markers of bone turnover for the prediction of fracture risk and monitoring of osteoporosis treatment: a need for international reference standards. Osteoporos Int. 2011 Feb;22(2):391-420.

24 Jørgensen HS, Winther S, Bøttcher M, Hauge EM, Rejnmark L, Svensson M, et al. Bone turnover markers are associated with bone density, but not with fracture in end stage kidney disease: a cross-sectional study. BMC Nephrol. 2017 Dec;18(1):284.

25 Bonani M, Frey D, Brockmann J, Fehr T, Mueller TF, Saleh L, et al. Effect of twice-yearly denosumab on prevention of bone mineral density loss in de novo kidney transplant recipients: a randomized controlled trial. Am J Transplant. 2016 Jun;16(6):1882-91.

26 Seibel MJ. Biochemical markers of bone turnover: part I: biochemistry and variability. Clin Biochem Rev. 2005 Nov;26(4):97-122.

27 Stokes FJ, Ivanov P, Bailey LM, Fraser WD. The effects of sampling procedures and storage conditions on shortterm stability of blood-based biochemical markers of bone metabolism. Clin Chem. 2011 Jan;57(1):138-40.

28 Bjarnason NH, Henriksen EE, Alexandersen P, Christgau S, Henriksen DB, Christiansen C. Mechanism of circadian variation in bone resorption. Bone. 2002 Jan;30(1):307-13.

29 Bonani M, Rodriguez D, Fehr T, Mohebbi N, Brockmann J, Blum M, et al. Sclerostin blood levels before and after kidney transplantation. Kidney Blood Press Res. 2014;39(4):230-9.

30 Harrell FEJ. With contributions from Charles Dupont and many others. 2019.

31 Wickham H. ggplot2. New York, NY: Springer New York; 2009.

32 Moe S, Drüeke T, Cunningham J, Goodman W, Martin K, Olgaard K, et al. Definition, evaluation, and classification of renal osteodystrophy: a position statement from Kidney Disease: Improving Global Outcomes (KDIGO). Kidney Int. 2006 Jun;69(11):1945-53.

33 Keronen S, Martola L, Finne P, Burton IS, Kröger H, Honkanen E. Changes in bone histomorphometry after kidney transplantation. Clin J Am Soc Nephrol. 2019 Jun;14(6):894-903.

34 Evenepoel P, Behets GJ, Viaene L, D’Haese PC. Bone histomorphometry in de novo renal transplant recipients indicates a further decline in bone resorption 1 year posttransplantation. Kidney Int. 2017 Feb;91(2):469-76.

35 Nickolas TL, Stein EM, Dworakowski E, Nishiyama KK, Komandah-Kosseh M, Zhang CA, et al. Rapid cortical bone loss in patients with chronic kidney disease. J Bone Miner Res. 2013 Aug;28(8):1811-20.

36 Toussaint ND, Lau KK, Strauss BJ, Polkinghorne KR, Kerr PG. Associations between vascular calcification, arterial stiffness and bone mineral density in chronic kidney disease. Nephrol Dial Transplant. 2007 Oct; 23(2):586-93.

37 Fürstenberg A, Buscombe J, Davenport A. Overestimation of lumbar spine calcium with dual energy x-ray absorptiometry scanning due to the prescription of lanthanum carbonate in patients with chronic kidney disease. Am J Nephrol. 2010;32(5):425-31.

38 Mann ML, Thornley-Brown D, Campbell R, Bell E, Burroughs L, Nunnally N, et al. The effect of peritoneal dialysate on DXA bone densitometry results in patients with end-stage renal disease. J Clin Densitom. 2008 Oct; 11(4):532-6.

39 Brown JP, Prince RL, Deal C, Recker RR, Kiel DP, de Gregorio LH, et al. Comparison of the effect of denosumab and alendronate on BMD and biochemical markers of bone turnover in postmenopausal women with low bone mass: a randomized, blinded, phase 3 trial. J Bone Miner Res. 2009 Jan;24(1):153-61. 